\title{
Association of EGFR mutations in second primary lung cancer and HER2 expression in breast cancer survivors
}

\author{
Yoon Jung Jang ${ }^{1 \#}$, Seo Yun Kim ${ }^{2 \#}$, Hong Kyu Jung ${ }^{1}$, Hye-Ryoun Kim ${ }^{2}$, Cheol Hyeon Kim ${ }^{2}$, Hyo-Rak Lee ${ }^{3}$, \\ Hye Jin Kang ${ }^{3}$, Sung Hyun Yang ${ }^{3}$, Hyesil Seol ${ }^{4}$, Im Il Na ${ }^{3} \wedge$ \\ ${ }^{1}$ Department of Internal Medicine, Korea Cancer Center Hospital, Korea Institute of Radiological Sciences, Seoul, Korea; ${ }^{2}$ Division of Pulmonary \\ and Critical Care Medicine, Korea Cancer Center Hospital, Korea Institute of Radiological Sciences, Seoul, Korea; ${ }^{3}$ Division of Hematology and \\ Medical Oncology, Korea Cancer Center Hospital, Korea Institute of Radiological Sciences, Seoul, Korea; ${ }^{4}$ Division of Pathology, Korea Cancer \\ Center Hospital, Korea Institute of Radiological Sciences, Seoul, Korea \\ Contributions: (I) Conception and design: SY Kim, HJ Kang, CH Kim, II Na; (II) Administrative support: HR Kim, HR Lee, SH Yang, H Seol; (III) \\ Provision of study materials or patients: HK Jung, HR Kim, H Seol; (IV) Collection and assembly of data: YJ Jang, HK Jung, HJ Kang, SH Yang; \\ (V) Data analysis and interpretation: YJ Jang, SY Kim, CH Kim, HR Lee, II Na; (VI) Manuscript writing: All authors; (VII) Final approval of the \\ manuscript: All authors. \\ \#These authors contributed equally to this work. \\ Correspondence to: Im Il Na, MD, PhD. Division of Hematology and Medical Oncology, Korea Cancer Center Hospital, Korea Institute of \\ Radiological Sciences, Nowon-gil, Nowon-gu, Seoul 139-70, Korea. Email: hmona71@gmail.com.
}

Background: The incidence of second primary lung cancer (SPLC) is increasing with longer survival rates from breast cancer. Despite of studies to suggest the mutual exclusivity of epidermal growth factor receptor (EGFR) and human epidermal growth receptor 2 (HER2) in several cancers, the effect of HER2 expression in breast cancer on EGFR mutations in SPLC is unclear. Therefore, this study aimed to determine the association between HER2 expression and EGFR mutations.

Methods: We conducted a retrospective cohort study of breast cancer survivors diagnosed with SPLC after breast cancer treatment between 1997 and 2018. We investigated the association between HER2 expression in breast cancer and EGFR mutations in SPLC, specifically focusing on negative correlations by using logistic regression analysis.

Results: EGFR mutations in SPLC were detected in 19 of 38 patients. Analysis for HER2 revealed a statistically significant difference in the proportion of EGFR mutations between patients with SPLC and previous HER2 positive breast cancer (43.5\%) and those with SPLC and previous HER2 negative breast cancer $(90.0 \% ; \mathrm{P}=0.021)$. The ratio of EGFR mutations decreased with the degree of HER2 expression in patients with previous breast cancer (90.0\% : for no HER2 expression, $62.5 \%$ for HER2 1+, 0.0\% for HER2 $2+$, and $41.7 \%$ for HER2 $3+; \mathrm{P}=0.018)$. Multivariate logistic analyses revealed that EGFR mutations in SPLC were significantly associated with age [odds ratio (OR): 1.11, 95\% confidence interval (CI): 1.01-0.23, $\mathrm{P}=0.039]$ and HER2 positive status (OR: 0.04, 95\% CI: 0.01-0.56, $\mathrm{P}=0.017$ ).

Conclusions: This study suggests that the frequency of EGFR mutations in SPLC may be associated with low HER2 expression in previous breast cancer.

Keywords: Breast cancer; lung cancer; HER2; EGFR; second primary cancer

Submitted Jul 09, 2021. Accepted for publication Nov 04, 2021.

doi: $10.21037 /$ tcr-21-1235

View this article at: https://dx.doi.org/10.21037/tcr-21-1235

^ ORCID: Yoon Jung Jang, 0000-0001-5186-136X; Im Il Na, 0000-0002-3872-6179. 


\section{Introduction}

In Korea, breast cancer, a common cancer affecting women for the past several decades, had the highest incidence in 2017 (1). The survival rates of patients with breast cancer have been increasing with early diagnosis and treatment improvement (2). Improved outcomes have led to an increased population of breast cancer survivors; hence, there has been a growing interest in determining the risk of second primary cancers $(3,4)$. In addition, second primary cancers are the cause of death in more than onehalf of breast cancer survivors (5). Numerous factors such as hereditary predisposition, common etiologic exposures, and especially the effects of chemotherapy or radiotherapy for the previous breast cancer may play an important role in the occurrence of second primary cancers (6-8). Lung cancer is the most common second primary cancer among breast cancer survivors (9). Previous studies have shown that approximately $5-10 \%$ of breast cancer patients are diagnosed with second primary cancers (5).

The incidence of second primary lung cancer (SPLC) is increasing with longer survival rates from breast cancer; however, only few studies have investigated the association between SPLC and breast cancer. In a large registry-based study, the incidence rate of SPLC was significantly higher in estrogen receptor (ER)-negative breast cancer patients than in ER-positive breast cancer patients (10). Another study reported that the incidence of SPLC was higher in human epidermal receptor 2 (HER2) negative breast cancer survivors than in HER2 positive breast cancer survivors. A recent retrospective study showed that the frequency of previous breast cancer was higher in patients with epidermal growth factor receptor (EGFR)-mutant lung cancer than in those with EGFR wild-type lung cancer (11). The family of EGFR receptors plays an important role in cell proliferation (12). However, interest has been centered on EGFR because EGFR mutations greatly affect the prognosis of lung cancer (13). HER2, another member of the EGFR family, is overexpressed in $15-25 \%$ of invasive breast cancers and is associated with reduced survival in invasive breast cancer (14). A few studies investigating the association between EGFR and HER2 in cancers have reported that EGFR and HER2 appear to be mutually exclusive in several cancers $(15,16)$. However, no study has investigated the effect of HER2 expression in breast cancer on EGFR mutations in SPLC.

In this study, we investigated the association between HER2 expression in breast cancer and EGFR mutations in
SPLC among breast cancer survivors, specifically focusing on negative correlations between HER2 status and EGFR mutation. We present the following article in accordance with the STROBE reporting checklist (available at https:// dx.doi.org/10.21037/tcr-21-1235).

\section{Methods}

\section{Patients and data collection}

This study included patients diagnosed with SPLC following breast cancer at the Korea Cancer Center Hospital (KCCH) between January 1997 and January 2018. Inclusion criteria were a pathological diagnosis of lung cancer and a previous history of primary breast cancer diagnosis in our hospital. Screening for SPLC was performed immediately after the diagnosis of breast cancer and included concurrent diagnosis of breast cancer and lung cancer. Finally, this study included patients with SPLC and previous breast cancer confirmed by pathologists at the KCCH. All pathological types of breast cancer were included, but small-cell lung cancer was excluded. Data of all patients were extracted from the Electronic Medical Record (EMR). Relevant clinical information [i.e., age, sex, current smoking status, pathological type of breast cancer and lung cancer, hormonal status of breast cancer (estrogen receptor and progesterone receptor), mutational status (HER2 expression in breast cancer and EGFR mutation in lung cancer), location of breast cancer, breast cancer treatment history, and initial clinical stage by tumornode-metastasis (TNM) seventh edition] were extracted from the EMR for analysis. The study was conducted in accordance with the Declaration of Helsinki (revised in 2013). The study was approved by the Institutional Review Board of Korea Cancer Center Hospital (2019-11-001), and individual consent for this retrospective analysis was waived.

\section{Immunobistochemistry and molecular analysis}

Samples of pathologically confirmed breast cancer tissues were immunostained with rabbit monoclonal antibody, 4B5, for HER2/neu using an automated staining system (Benchmark XT, Ventana Medical Systems, Tucson Arizona, USA) according to the manufacturer's recommendations. Staining for HER2 was detected using the OptiView DAB IHC detection kit (Ventana Medical Systems, Tucson Arizona, USA) and scored from 0 to 3 using the scoring 

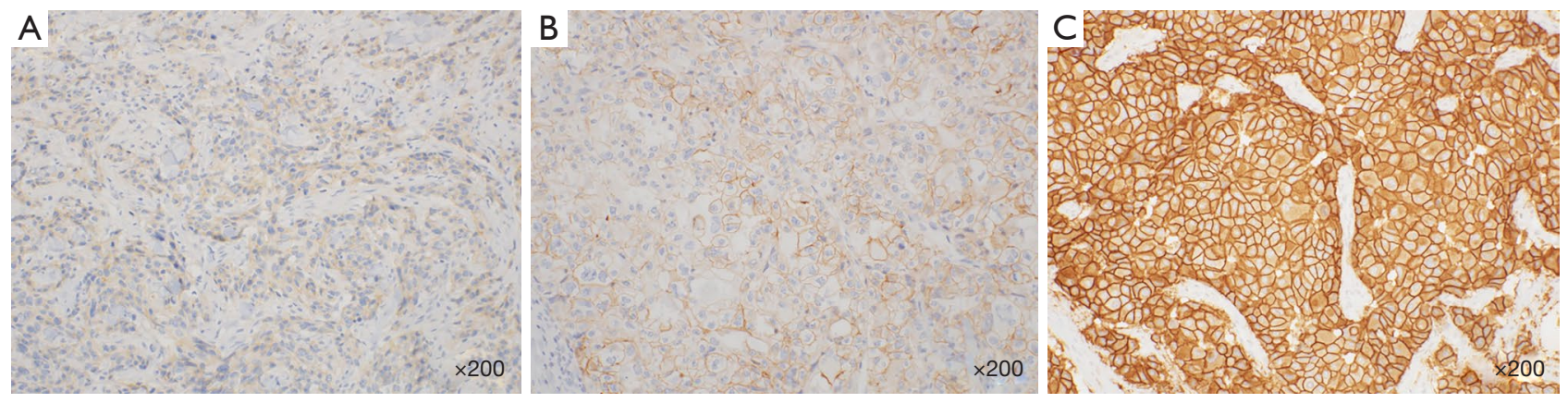

Figure 1 Images $(\times 200)$ of immunohistochemical staining for HER2 in breast cancer samples. Staining performed using rabbit monoclonal antibody for HER2/neu and detected using the OptiView DAB IHC detection Kit. (A) 1+ HER2 expression (B) 2+ HER2 expression (C) 3+ HER2 expression. HER2, human epidermal growth receptor 2.

system reported in the guidelines (Figure 1) (17). Samples with a HER2 score of $1+$ to $3+$ were defined as HER2 positive. Estrogen and progesterone receptors were evaluated according to the American Society of Clinical Oncology/College of American Pathologists guidelines (18). Genomic DNA from tumor tissue samples were analyzed for EGFR mutations using the PyroMark Q96 ID PCR kit (Qiagen, Valencia, CA, USA) and an ABI 3130 analyzer (Thermo Fisher Scientific, Foster City, CA, USA). The samples were analyzed for point mutations and in-frame deletions in exons $18,19,20$, and 21 , as recommended by the Human Genome Variation Society.

\section{Statistical analysis}

Categorical variables are presented as number (percentage), and continuous variables are presented as median [interquartile range (IQR)]. The association between HER2 expression and EGFR mutations was assessed using Fisher's exact test. In addition, the linear-by-linear association test was used to analyze trends in EGFR mutation according to the degree of HER2 expression. Univariate and multivariate logistic regression analyses were performed to identify risk factors associated with EGFR mutations. Covariates with a $\mathrm{P}$ value of $<0.1$ in the univariate analysis were included in the multivariate logistic regression analysis with the stepwise method using the backward selection approach. Considering our small sample size, the adjusted multivariate model was evaluated using the goodness-of-fit, the pseudo R-square, and the likelihood ratio. A $\mathrm{P}$ value of $<0.05$ was considered statistically significant. All $\mathrm{P}$ values reported were two-sided. Odds ratios (ORs) and 95\% confidence intervals (CIs) were calculated for each factor. Statistical analyses were performed using Statistical Package for Social Science (SPSS version 24.0 for Windows; SPSS, Chicago, USA).

\section{Results}

A total of 38 patients with SPLC and previous breast cancer were identified. Patient characteristics are presented in Table 1. All patients were women, with a median age of 65 years (IQR, 58.0-74.5 years). The majority of patients with previous breast cancer $(81.6 \%)$ had invasive ductal carcinoma. Thirty-three (86.8\%) patients were evaluated for HER2 expression, of which 23 (69.7\%) patients were HER2 positive. Nearly all (97.4\%) patients underwent surgery for breast cancer treatment, of which approximately half $(55.3 \%)$ received radiotherapy. A major pathological feature of SPLCs was adenocarcinoma (86.8\%). EGFR mutations were confirmed in $19(50 \%)$ patients [3 (15.8\%) patients: exon 18; $6(31.6 \%)$ patients: exon 19; and 10 (52.6\%) patients: exon 21].

\section{Correlation between the degree of HER2-positive expression in breast cancer and EGFR mutation in lung cancer}

HER2 expression data of five patients were not available. The results of HER2 expression analysis in the remaining 33 patients are presented in Figure 2. Eight (24.2\%) patients had a HER2 score of 1+, 3 (9.1\%) had 2+, and 12 (36.4\%) had $3+$. EGFR mutation showed a negative association with HER2 expression (90\% in HER2 negative vs. 43.5\% in HER2 positive, $\mathrm{P}=0.021$ ). In addition, EGFR mutation tended to decrease with an increase in HER2 expression $(\mathrm{P}=0.018)$. 
Table 1 Patient characteristics

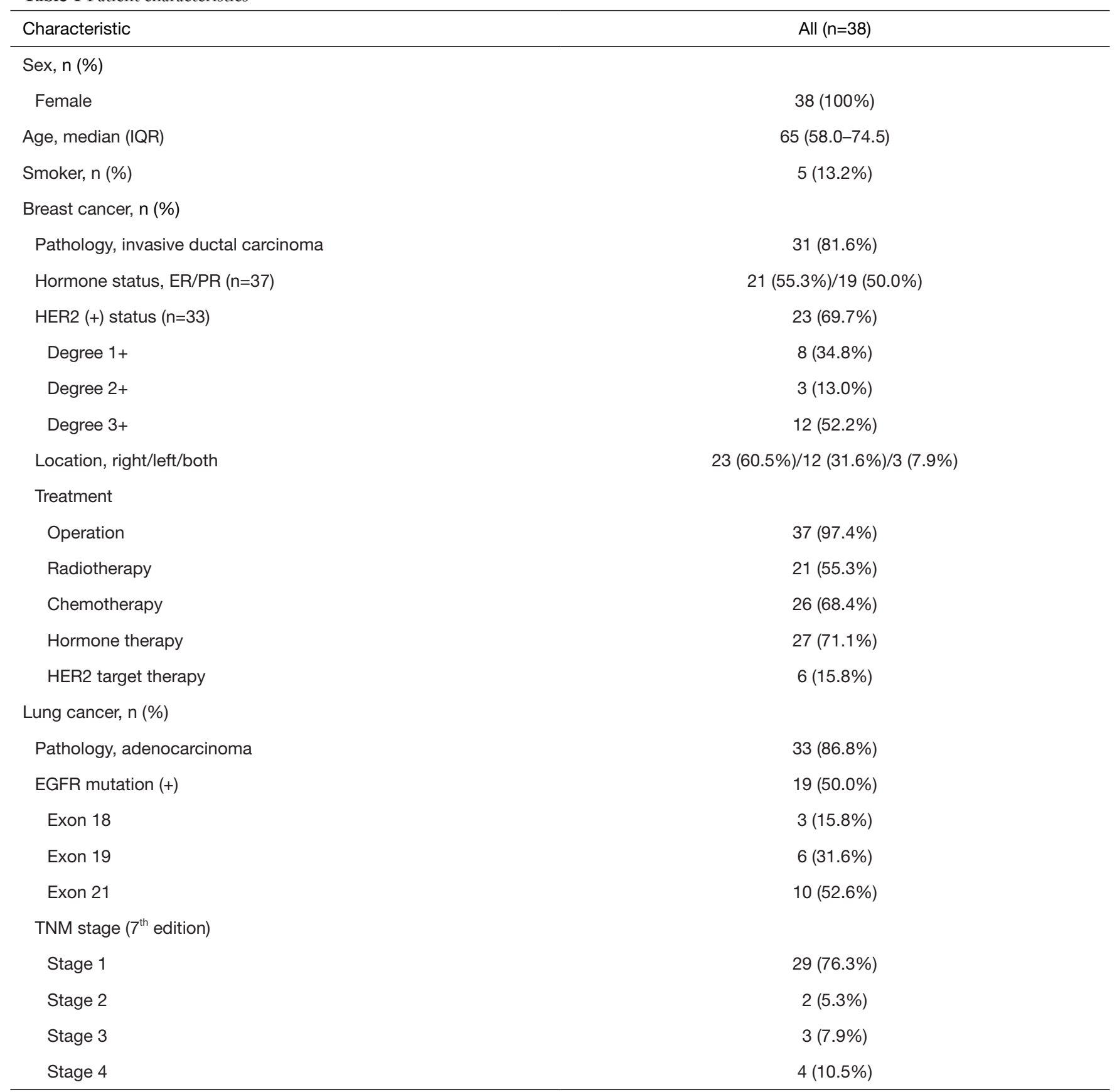

Results are reported as $\mathrm{n}(\%)$ or median (IQR). No, number; IQR, interquartile range; ER, estrogen receptor; PR, progesterone receptor; HER2, human epidermal receptor 2; EGFR, epidermal growth factor receptor; TNM, tumour, node, metastasis.

Comparison of EGFR mutation risk in the univariate and multivariate model

The independent effects of covariates on the risk of EGFR mutations are shown in Table 2. In the univariate analysis, HER2 expression (OR: 0.085, 95\% CI: 0.01-0.79, P=0.03) and previous hormonal therapy (OR: 7.44, 95\% CI: $1.25-$ 44.19, $\mathrm{P}=0.027$ ) were associated with the risk of EGFR mutations. After adjusting for age, estrogen receptor status, and HER2 expression, HER2 expression was associated with a decreased risk of EGFR mutations (OR: 0.04, 95\% 


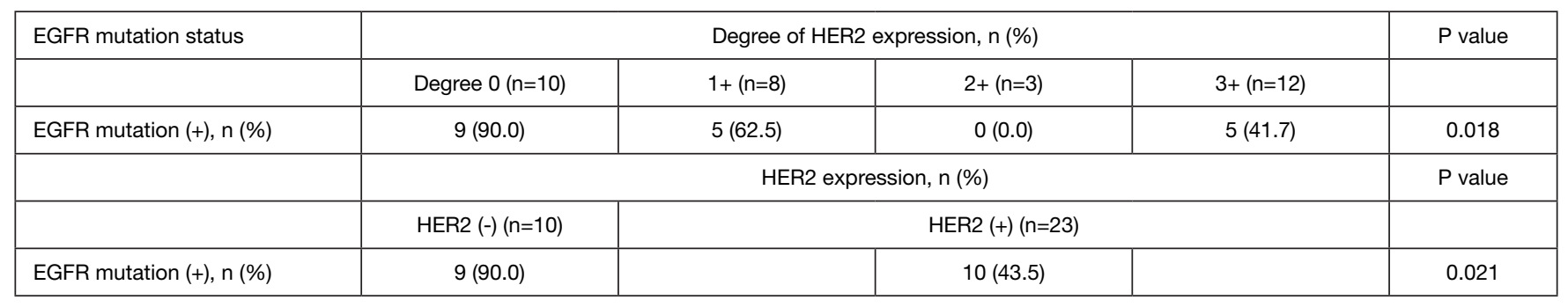

Figure 2 Trend test for EGFR mutations in lung cancer according to the status of HER2 expression in breast cancer. Results are reported as n (\%). HER2, human epidermal receptor 2; EGFR, epidermal growth factor receptor.

Table 2 Univariable and multivariable analyses of risk factors associated with EGFR mutations

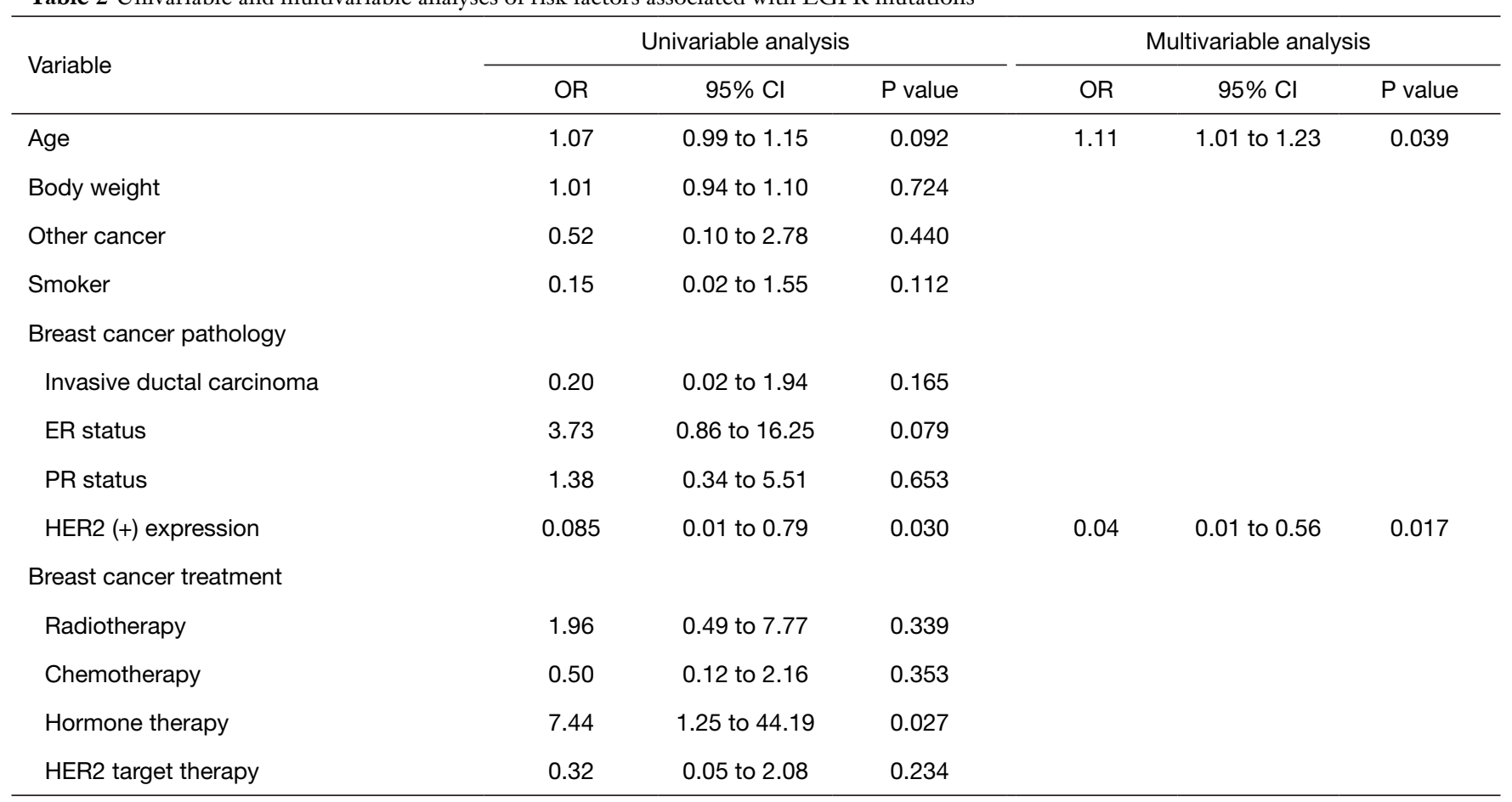

EGFR, epidermal growth factor receptor; ER, estrogen receptor; PR, progesterone receptor; HER2, human epidermal receptor 2; OR, odds ratio; $\mathrm{Cl}$, confidence interval.

CI: $0.01-0.56, \mathrm{P}=0.017)$ in the multivariate analysis. In addition, the risk of EGFR mutation increased with age (OR 1.11, 95\% CI: 1.01-1.23, $\mathrm{P}=0.039)$. The logistic regression model was statistically significant $\left\{\chi^{2}[2]=12.39 ; \mathrm{P}=0.002\right\}$. The final model explained $42.1 \%$ (Nagelkerke $\mathrm{R}^{2}$ ) of the variance in EGFR mutations and correctly classified $75.8 \%$ of cases.

\section{Discussion}

This study suggests that the risk of developing EGFR- mutant SPLCs may be significantly lower in patients with previous HER2 positive breast cancer than in those with HER2 negative breast cancer.

The correlation between HER2 status in breast cancer and EGFR-mutant SPLCs has not been reported previously. However, many previous studies have reported the elevated risk for second primary cancers, such as lung cancer after breast cancer, suggesting that treatments such as radiotherapy might be associated with increased risks (19-21). In addition, most studies on the association between EGFR mutations and HER2 overexpression have 
focused on the same tumor tissue (22-24). Few studies have demonstrated the risk of developing a second primary cancer based on the status of the initial cancer. For example, a study reported that the risk of developing an SPLC after invasive breast cancer varied with estrogen receptor status (10). Another single-center, retrospective study indicated that a higher proportion of patients with EGFR-mutant lung cancer had previous breast cancer compared to patients with EGFR non-mutant lung cancer (11). However, the association between HER2 overexpression and EGFR mutations was not analyzed in this study. To the best of our knowledge, our study is the first to demonstrate that the HER2 status in previous breast cancer might induce the occurrence of SPLC with EGFR mutation.

Although several factors may contribute to the increased risk of EGFR mutation in SPLC after HER2 negative breast cancer, the concept of oncogene addiction might explain this result $(25,26)$. High dependence of cancer cells on a certain oncogene during development causes other oncogenes to lose their ability to proliferate (26). For example, EGFR mutations are considered to be mutually exclusive with echinoderm microtubule-associated proteinlike 4-anaplastic lymphoma kinase rearrangement, except in rare concomitant cases $(27,28)$. In line with this theory, previous breast cancers with HER2 overexpression might be mutually exclusive with the activation of other oncogenes. Therefore, a patient with previous HER2 positive breast cancer may not develop EGFR-mutant lung cancer.

In the present study, the rate of EGFR mutations in lung cancer increased with age. Previous studies have consistently reported positive associations between EGFR mutations and age (29). One retrospective study showed that compared to older patients, fewer patients aged $\leq 50$ years had EGFR mutations (30). Other studies have suggested a significant increase in the prevalence of EGFR mutations with an increased age in female patients who have never smoked (31). A possible mechanism is that EGFR mutations accumulate with increasing age in patients with lung cancer, resulting in carcinogenesis. Larger studies are required to elucidate other possible mechanisms. In addition, smoking was not found to be a risk factor for EGFR mutations in the multivariate analysis of this study. Similarly, studies have reported that most EGFR-mutant tumors are found in 'never smokers', and that the number of smoking pack-years predicts the prevalence of EGFR mutations (32,33).

HER2 overexpressing breast cancers have different characteristics compared with HER2 negative breast cancers. HER2 overexpressing breast cancers are more likely to have an aggressive tumor grade, a higher risk of recurrence, and a relatively high incidence of brain metastasis than HER2 negative breast cancers (34). However, no remarkable risk factors for HER2 positive breast cancers have been found. Few studies have reported the etiology of HER2 overexpression. A study conducted in China indicated that parity and breastfeeding were inversely associated with HER2 positive breast cancer (35). In addition, other studies have found that older age at first birth or younger age at menarche might affect HER2 overexpression (36,37).

Several methodological limitations should be considered when interpreting the results of this study. First, this study included a small number of patients and therefore, it is difficult to make statistically significant judgments. Only three patients had a HER2 score of $2+$ without EGFR mutations. However, despite the small sample size of the study, a strong association between HER2 expression and EGFR mutation was found consistently in both the trend test and multivariate analysis. Second, individual risk factors for EGFR mutations, rather than HER2 overexpression alone, might skew these results. Particularly, EGFR mutations usually develop among Asian, female, and nonsmoker populations (38). Nevertheless, the association we observed is worth considering because our study population was mostly homogeneous in terms of patient characteristics (such as age, ethnicity, sex, and smoking history) and thus, had similar risk factors for EGFR mutations. Finally, detailed data on prognosis were not available. The presence of EGFR mutations in SPLC is expected to affect clinical outcomes. Further studies evaluating the prognosis of lung cancer after breast cancer are warranted.

\section{Conclusions}

The results of the current study suggest that EGFR mutations in SPLC are more likely to develop in HER2 negative breast cancer survivors. With advances in breast cancer treatment, the number of breast cancer survivors is increasing. Therefore, further studies investigating whether the higher expression of HER2 in previous breast cancer results in an increased risk of developing SPLC without EGFR mutations.

\section{Acknowledgments}

We would like to thank Editage (www.editage.co.kr) for English language editing.

Funding: None. 


\section{Footnote}

Reporting Checklist: The authors have completed the STROBE reporting checklist. Available at https://dx.doi. org/10.21037/tcr-21-1235

Data Sharing Statement: Available at https://dx.doi. org/10.21037/tcr-21-1235

Conflicts of Interest: All authors have completed the ICMJE uniform disclosure form (available at https://dx.doi. org/10.21037/tcr-21-1235). The authors have no conflicts of interest to declare.

Ethical Statement: The authors are accountable for all aspects of the work in ensuring that questions related to the accuracy or integrity of any part of the work are appropriately investigated and resolved. The study was conducted in accordance with the Declaration of Helsinki (revised in 2013). The study was approved by the Institutional Review Board of Korea Cancer Center Hospital (2019-11-001), and individual consent for this retrospective analysis was waived.

Open Access Statement: This is an Open Access article distributed in accordance with the Creative Commons Attribution-NonCommercial-NoDerivs 4.0 International License (CC BY-NC-ND 4.0), which permits the noncommercial replication and distribution of the article with the strict proviso that no changes or edits are made and the original work is properly cited (including links to both the formal publication through the relevant DOI and the license). See: https://creativecommons.org/licenses/by-nc-nd/4.0/.

\section{References}

1. Hong S, Won YJ, Park YR, et al. Cancer Statistics in Korea: Incidence, Mortality, Survival, and Prevalence in 2017. Cancer Res Treat 2020;52:335-50.

2. Park EH, Min SY, Kim Z, et al. Basic Facts of Breast Cancer in Korea in 2014: The 10-Year Overall Survival Progress. J Breast Cancer 2017;20:1-11.

3. Schaapveld M, Visser O, Louwman MJ, et al. Risk of new primary nonbreast cancers after breast cancer treatment: a Dutch population-based study. J Clin Oncol 2008;26:1239-46.

4. Kang SY, Lee SB, Kim YS, et al. Breast Cancer Statistics in Korea, 2018. J Breast Cancer 2021;24:123-37.
5. Donin N, Filson C, Drakaki A, et al. Risk of second primary malignancies among cancer survivors in the United States, 1992 through 2008. Cancer 2016;122:3075-86.

6. Neugut AI, Robinson E, Lee WC, et al. Lung cancer after radiation therapy for breast cancer. Cancer 1993;71:3054-7.

7. Rubino C, de Vathaire F, Diallo I, et al. Increased risk of second cancers following breast cancer: role of the initial treatment. Breast Cancer Res Treat 2000;61:183-95.

8. Roychoudhuri R, Evans H, Robinson D, et al. Radiationinduced malignancies following radiotherapy for breast cancer. Br J Cancer 2004;91:868-72.

9. Ko KH, Huang HK, Chen YI, et al. Surgical outcomes of second primary lung cancer after the extrapulmonary malignancy. J Cancer Res Clin Oncol 2020;146:3323-32.

10. Schonfeld SJ, Curtis RE, Anderson WF, et al. The risk of a second primary lung cancer after a first invasive breast cancer according to estrogen receptor status. Cancer Causes Control 2012;23:1721-8.

11. Moran T, Quiroga V, Cirauqui B, et al. A Single-Center Retrospective Study of Patients with Double Primary Cancers: Breast Cancer and EGFR-Mutant Non-Small Cell Lung Cancer. Oncol Res Treat 2019;42:107-14.

12. Han W, Lo HW. Landscape of EGFR signaling network in human cancers: biology and therapeutic response in relation to receptor subcellular locations. Cancer Lett 2012;318:124-34.

13. Ali R, Wendt MK. The paradoxical functions of EGFR during breast cancer progression. Signal Transduct Target Ther 2017;2:16042.

14. Sirkisoon SR, Carpenter RL, Rimkus T, et al. EGFR and HER2 signaling in breast cancer brain metastasis. Front Biosci (Elite Ed) 2016;8:245-63.

15. Takezawa K, Pirazzoli V, Arcila ME, et al. HER2 amplification: a potential mechanism of acquired resistance to EGFR inhibition in EGFR-mutant lung cancers that lack the second-site EGFRT790M mutation. Cancer Discov 2012;2:922-33.

16. Crasta J, Ravikumar G, Rajarajan S, et al. Expression of HER2 and EGFR Proteins in Advanced Stage High-grade Serous Ovarian Tumors Show Mutual Exclusivity. Int J Gynecol Pathol 2021;40:49-55.

17. Hofmann M, Stoss O, Shi D, et al. Assessment of a HER2 scoring system for gastric cancer: results from a validation study. Histopathology 2008;52:797-805.

18. Hammond ME, Hayes DF, Wolff AC, et al. American society of clinical oncology/college of american 
pathologists guideline recommendations for immunohistochemical testing of estrogen and progesterone receptors in breast cancer. J Oncol Pract 2010;6:195-7.

19. Lorigan P, Califano R, Faivre-Finn C, et al. Lung cancer after treatment for breast cancer. Lancet Oncol 2010;11:1184-92.

20. Zablotska LB, Neugut AI. Lung carcinoma after radiation therapy in women treated with lumpectomy or mastectomy for primary breast carcinoma. Cancer 2003;97:1404-11.

21. Clarke M, Collins R, Darby S, et al. Effects of radiotherapy and of differences in the extent of surgery for early breast cancer on local recurrence and 15-year survival: an overview of the randomised trials. Lancet 2005;366:2087-106.

22. Lee HJ, Seo AN, Kim EJ, et al. Prognostic and predictive values of EGFR overexpression and EGFR copy number alteration in HER2-positive breast cancer. Br J Cancer 2015;112:103-11.

23. Pillai RN, Behera M, Berry LD, et al. HER2 mutations in lung adenocarcinomas: A report from the Lung Cancer Mutation Consortium. Cancer 2017;123:4099-105.

24. Ise N, Omi K, Nambara D, et al. Overexpressed HER2 in NSCLC is a possible therapeutic target of EGFR inhibitors. Anticancer Res 2011;31:4155-61.

25. Weinstein IB, Joe A. Oncogene addiction. Cancer Res 2008;68:3077-80; discussion 3080.

26. Ferrara MG, Di Noia V, D'Argento E, et al. OncogeneAddicted Non-Small-Cell Lung Cancer: Treatment Opportunities and Future Perspectives. Cancers (Basel) 2020;12:1196.

27. Gainor JF, Varghese AM, Ou SH, et al. ALK rearrangements are mutually exclusive with mutations in EGFR or KRAS: an analysis of 1,683 patients with nonsmall cell lung cancer. Clin Cancer Res 2013;19:4273-81.

28. Fan J, Dai X, Wang Z, et al. Concomitant EGFR Mutation and EML4-ALK Rearrangement in Lung Adenocarcinoma Is More Frequent in Multifocal Lesions. Clin Lung Cancer 2019;20:e517-30.

Cite this article as: Jang YJ, Kim SY, Jung HK, Kim HR, Kim CH, Lee HR, Kang HJ, Yang SH, Seol H, Na II. Association of EGFR mutations in second primary lung cancer and HER2 expression in breast cancer survivors. Transl Cancer Res 2021;10(12):5204-5211. doi: 10.21037/tcr-21-1235
29. Choi YH, Lee JK, Kang HJ, et al. Association between age at diagnosis and the presence of EGFR mutations in female patients with resected non-small cell lung cancer. J Thorac Oncol 2010;5:1949-52.

30. Wu SG, Chang YL, Yu CJ, et al. Lung adenocarcinoma patients of young age have lower EGFR mutation rate and poorer efficacy of EGFR tyrosine kinase inhibitors. ERJ Open Res 2017;3:00092-2016.

31. Ueno T, Toyooka S, Suda K, et al. Impact of age on epidermal growth factor receptor mutation in lung cancer. Lung Cancer 2012;78:207-11.

32. Pham D, Kris MG, Riely GJ, et al. Use of cigarettesmoking history to estimate the likelihood of mutations in epidermal growth factor receptor gene exons 19 and 21 in lung adenocarcinomas. J Clin Oncol 2006;24:1700-4.

33. Pao W, Miller V, Zakowski M, et al. EGF receptor gene mutations are common in lung cancers from "never smokers" and are associated with sensitivity of tumors to gefitinib and erlotinib. Proc Natl Acad Sci U S A 2004;101:13306-11.

34. Iqbal N, Iqbal N. Human Epidermal Growth Factor Receptor 2 (HER2) in Cancers: Overexpression and Therapeutic Implications. Mol Biol Int 2014;2014:852748.

35. Xing P, Li J, Jin F. A case-control study of reproductive factors associated with subtypes of breast cancer in Northeast China. Med Oncol 2010;27:926-31.

36. Phipps AI, Buist DS, Malone KE, et al. Reproductive history and risk of three breast cancer subtypes defined by three biomarkers. Cancer Causes Control 2011;22:399-405.

37. Phipps AI, Malone KE, Porter PL, et al. Reproductive and hormonal risk factors for postmenopausal luminal, HER2-overexpressing, and triple-negative breast cancer. Cancer 2008;113:1521-6.

38. Gaur P, Bhattacharya S, Kant S, et al. EGFR Mutation Detection and Its Association With Clinicopathological Characters of Lung Cancer Patients. World J Oncol 2018;9:151-5. 